\title{
The Mahler measure of a Weierstrass form
}

\author{
Matilde N. Lalín* and Frank Ramamonjisoa ${ }^{\dagger}$ \\ Département de Mathématique et de Statistique \\ Université de Montréal \\ CP 6128, succ. Centre-Ville, Montreal \\ QC, Canada H3C 3J7 \\ *mlalin@dms.umontreal.ca \\ †rank.ramamonjisoa@umontreal.ca
}

Received 27 July 2016

Accepted 19 October 2016

Published 4 April 2017

\begin{abstract}
We prove an identity between Mahler measures of polynomials that was originally conjectured by Boyd. The combination of this identity with a result of Zudilin leads to a formula involving a Mahler measure of a Weierstrass form of conductor 17 given in terms of $L^{\prime}(E, 0)$. Our proof involves a non-trivial identity between regulators which leads to the elliptic curve $L$-function being expressed in terms of the regulator evaluated in a non-rational non-torsion point.
\end{abstract}

Keywords: Mahler measure; special values of $L$-functions; elliptic curve; elliptic regulator.

Mathematics Subject Classification 2010: 11R06, 11F67, 11G05, 11G16, 19F27, 33C75, $33 \mathrm{E} 05$

\section{Introduction}

For a non-zero multivariate rational function $P \in \mathbb{C}\left(x_{1}, \ldots, x_{n}\right)$, the (logarithmic) Mahler measure is defined as

$$
\mathrm{m}(P):=\frac{1}{(2 \pi i)^{n}} \int_{\mathbb{T}^{n}} \log \left|P\left(x_{1}, \ldots, x_{n}\right)\right| \frac{d x_{1}}{x_{1}} \cdots \frac{d x_{n}}{x_{n}},
$$

where $\mathbb{T}^{n}=\left\{\left(x_{1}, \ldots, x_{n}\right) \in \mathbb{C}^{n}|| x_{1}|=\cdots=| x_{n} \mid=1\right\}$.

Boyd [7] systematically examined families of polynomials associated to elliptic curves and found numerical connections between their Mahler measures and special values of their $L$-functions. For example, Boyd considered the following two-variable

This is an Open Access article published by World Scientific Publishing Company. It is distributed under the terms of the Creative Commons Attribution 4.0 (CC-BY) License. Further distribution of this work is permitted, provided the original work is properly cited. 
families

$$
\begin{aligned}
& P_{k}(x, y):=x+\frac{1}{x}+y+\frac{1}{y}+k, \\
& F_{k}(x, y):=y^{2}+k x y-x^{3}-x,
\end{aligned}
$$

where $k$ is a parameter.

For $k \neq 0, \pm 4$, the zero set $P_{k}(x, y)=0$ is a genus-one curve $E_{N(k)}$, where $N(k)$ denotes the conductor. The same applies to the zero set $F_{k}(x, y)=0$ which is already given in Weierstrass form.

Boyd found for $k$ integral many formulas of the form

$$
\mathrm{m}\left(P_{k}\right) \stackrel{?}{=} r_{k} L^{\prime}\left(E_{N(k)}, 0\right),
$$

where $r_{k}$ is a rational number of low height and the question mark stands for a numerical formula that is true for at least 20 decimal places. He found similar formulas when $P_{k}$ is replaced by $F_{k}$ as well as several other one-parameter polynomial families.

Some equations of the type (1.1) were explained by Deninger [11] in terms of Beylinson's conjectures. Rodriguez-Villegas further investigated this connection and proved some of these formulas involving $P_{k}(x, y)$ for cases where the elliptic curve has complex multiplication and $k^{2} \in \mathbb{Z}$ (for instance, $k=4 \sqrt{2}$ ). In fact, it should be noted that the condition that $k$ is integral may be relaxed in several cases. For example, for the family $P_{k}(x, y)$, Boyd also found formulas of the type (1.1) for $k \in i \mathbb{Z}$.

After the complex multiplication cases, Rodriguez-Villegas [18] proved an identity involving two Mahler measures of Weierstrass forms coming from Boyd's conjectures, namely,

$$
7 \mathrm{~m}\left(y^{2}+2 x y+y-x^{3}-2 x^{2}-x\right)=5 \mathrm{~m}\left(y^{2}+4 x y+y-x^{3}+x^{2}\right) .
$$

In the formula above, each side is conjectured to be equal to $35 L^{\prime}\left(E_{37}, 0\right)$, where $E_{37}$ is the elliptic curve $E_{37}: y^{2}+y=x^{3}-x$ of conductor 37. Both Weierstrass forms involved in (1.2) are isomorphic to $E_{37}$. The technique for this proof is to relate the Mahler measure to the regulator within the framework of Bellinson's conjectures and then to prove the identity at the level of the regulator.

Following Rodriguez-Villegas' ideas, Bertin [1] proved

$$
\mathrm{m}\left(y^{2}-x y+y-x^{3}\right)=2 \mathrm{~m}\left((x+1) y^{2}+\left(x^{2}+x+1\right) y+x(x+1)\right) .
$$

In this formula, a Weierstrass form is related to a non-Weierstrass form, and both sides are expected to be equal to $2 L^{\prime}\left(E_{14}, 0\right)$. Further identities of this type were proven by Bertin [1, 2], Touafek and Kedara [21], Lalín and Rogers [15], Lalín [13], and Guillera and Rogers [12] by similar methods. An analogous identity involving polynomials conjecturally related to $L^{\prime}(E,-1)$ was proven by Lalín [14].

An important breakthrough in the area happened when formulas of the type (1.1) were proven for curves without complex multiplication. This involves the 
results of Brunault [8,9] and Mellit [16], who related the right-hand side of (1.3) to $2 L^{\prime}\left(E_{14}, 0\right)$, thus providing the first fully proven Mahler measure formula involving a Weierstrass form. Rogers and Zudilin [19, 20] proved several formulas including the original conjecture appearing both in [7] and [11], namely,

$$
\mathrm{m}\left(P_{1}\right)=L^{\prime}\left(E_{15}, 0\right) .
$$

Further breakthroughs came with a result due to Mellit, Brunault, and Zudilin [24] providing a systematic method of proof for some of these type of formulas involving modular unit parametrizations. This allowed many proofs and reproofs of identities involving the specific family $P_{k}(x, y)$. Further extensions of this method by Brunault [10] led to proofs of identities such as

$$
\mathrm{m}\left(y^{2}+k x y+y-x^{3}\right)=L^{\prime}\left(E_{N(k)}, 0\right)
$$

for $k=-2,-3$ where $E_{N(k)}$ has conductor 35,54 respectively.

In this work, we will prove the following result.

Theorem 1. We have the following identity between Mahler measures:

$$
4 \mathrm{~m}\left(F_{3}\right)=7 \mathrm{~m}\left(P_{i}\right) .
$$

Comparing this with the following result by Zudilin [24]

$$
\mathrm{m}\left(P_{i}\right)=2 L^{\prime}\left(E_{17}, 0\right),
$$

allows us to conclude the following identity.

Corollary 2. We have the following Mahler measure formula involving a Weierstrass form:

$$
\mathrm{m}\left(y^{2}+3 x y-x^{3}-x\right)=\frac{7}{2} L^{\prime}\left(E_{17}, 0\right) .
$$

To our knowledge, the result of Corollary 2 represents the first fully proven Mahler measure formula in the family $F_{k}$, and it is only the fourth Weierstrass form whose Mahler measure has been proven with the other three given by Eqs. (1.4) for $k=-1,-2,-3$.

In order to prove Theorem 1 we return to the methods used to prove (1.2) by relating the regulators. It should be noted that the identity between the regulators in this case is highly non-trivial, and considerably more involved than in any previous identities that were proven. In its simpler form, the regulator identity reads as

$$
7 D^{E}(P)=-4 D^{E}(A)
$$

where $P$ is a rational torsion point of order 4 while $A$ is a non-rational point of infinite order, which seems to go beyond some of the predictions for Beŭlinson's conjectures. We remark that the only cases among those mentioned above involving non-torsion points are both sides of (1.2), and even in this case, the involved points are rational. 
This article is organized as follows. In Sec. 2 we describe the theoretical framework connecting the Mahler measure formulas discussed in this article to the Berlinson regulator. In Sec. 3 we give the isomorphism relating the curves and some notable points in $E$, which we later use in Sec. 4 to establish the relationship between the regulators. In Sec. 5 we take a small detour from the proof to reflect on formula (1.7) in the context of Berlinson's conjectures. Then in Sec. 6 we characterize the homology class corresponding to the integration paths. We strive to include all the details of this computation, which is not always clear in previous works. Finally, we conclude the proof in Sec. 7 and comment on future directions in Sec. 8.

\section{Mahler Measure and the Regulator}

In this section we will recall the definition given by Bloch and Beullinson of the regulator map on the second $K$-group of an elliptic curve $E$ and explain how it can be computed in terms of the elliptic dilogarithm and how it can be related to the Mahler measure. We will start by giving all the elements involved in the definition of the regulator.

Let $F$ be a field. The second $K$-group of $F$ has a particularly simple expression given by Matsumoto's theorem as

$$
K_{2}(F) \cong \Lambda^{2} F^{\times} /\{x \otimes(1-x): x \in F, x \neq 0,1\} .
$$

Let $E / \mathbb{Q}$ be an elliptic curve. Under certain conditions that are verified in all the cases considered in this article (the triviality of tame symbols, see [17]), we can think of $K_{2}(E) \otimes \mathbb{Q} \subset K_{2}(\mathbb{Q}(E)) \otimes \mathbb{Q}$.

Let $x, y$ be two functions in $\mathbb{Q}(E)$. Then consider the differential form

$$
\eta(x, y):=\log |x| d \arg y-\log |y| d \arg x,
$$

where $d \arg x$ is defined by $\operatorname{Im}(d x / x)$.

The form $\eta(x, y)$ is multiplicative, antisymmetric, and satisfies

$$
\eta(x, 1-x)=d D(x),
$$

where $D(x)$ is the Bloch-Wigner dilogarithm given by

$$
D(x)=\operatorname{Im}\left(\operatorname{Li}_{2}(x)\right)+\arg (1-x) \log |x|,
$$

and

$$
\operatorname{Li}_{2}(x)=-\int_{0}^{x} \frac{\log (1-z)}{z} d z .
$$

We are now ready to give the main definition in this section.

Definition 3. The regulator map of Bloch [5] and Beulinson [3] is given by

$$
\begin{aligned}
r: K_{2}(E) \otimes \mathbb{Q} & \rightarrow H^{1}(E, \mathbb{R}) \\
\{x, y\} & \rightarrow\left\{\gamma \rightarrow \int_{\gamma} \eta(x, y)\right\} .
\end{aligned}
$$


Remark 4. Notice the following facts.

- The regulator is actually defined over $K_{2}(\mathcal{E})$, where $\mathcal{E}$ is the Néron model of the elliptic curve. $K_{2}(\mathcal{E}) \otimes \mathbb{Q}$ is a subgroup of $K_{2}(E) \otimes \mathbb{Q}$ determined by finitely many extra conditions (see $[6]$ ).

- In the above definition, we take $\gamma \in H_{1}(E, \mathbb{Z})$ and interpret $H^{1}(E, \mathbb{R})$ as the dual of $H_{1}(E, \mathbb{Z})$.

- Because of the way that complex conjugation acts on $\eta$, the regulator map is trivial for the classes in $H_{1}(E, \mathbb{Z})^{+}$, the cycles that remain invariant by complex conjugation. Therefore it suffices to consider the regulator as a function on $H_{1}(E, \mathbb{Z})^{-}$.

We will now explain how to compute the integral of $\eta(x, y)$. Recall that $E$ is an elliptic curve defined over $\mathbb{Q}$. Then we can write

$$
\begin{gathered}
E(\mathbb{C}) \stackrel{\sim}{\rightarrow} \mathbb{C} /(\mathbb{Z}+\tau \mathbb{Z}) \stackrel{\sim}{\rightarrow} \mathbb{C}^{\times} / q^{\mathbb{Z}} \\
S=\left(\wp(u), \wp^{\prime}(u)\right) \rightarrow u \bmod \Lambda \rightarrow z=e^{2 \pi i u}
\end{gathered}
$$

where $\wp$ is the Weierstrass function, $\Lambda$ is the lattice $\mathbb{Z}+\tau \mathbb{Z}, \tau \in \mathbb{H}$, and $q=e^{2 \pi i \tau}$. The next definition is due to Bloch [5].

Definition 5. The elliptic dilogarithm is a function on $E(\mathbb{C})$ given for $S \in E(\mathbb{C})$ by

$$
D^{E}(S)=\sum_{n \in \mathbb{Z}} D\left(q^{n} z\right),
$$

where $D$ is the Bloch-Wigner dilogarithm defined by $(2.1)$.

Let $\mathbb{Z}[E(\mathbb{C})]$ be the group of divisors on $E$ and let

$$
\mathbb{Z}[E(\mathbb{C})]^{-} \cong \mathbb{Z}[E(\mathbb{C})] /\{(S)+(-S): S \in E(\mathbb{C})\} .
$$

Let $x, y \in \mathbb{C}(E)^{\times}$. The diamond operation is defined by

$$
\begin{aligned}
\diamond: \Lambda^{2} \mathbb{C}(E)^{\times} & \rightarrow \mathbb{Z}[E(\mathbb{C})]^{-} \\
(x) \diamond(y) & =\sum_{i, j} m_{i} n_{j}\left(S_{i}-T_{j}\right),
\end{aligned}
$$

where

$$
(x)=\sum_{i} m_{i}\left(S_{i}\right) \quad \text { and } \quad(y)=\sum_{j} n_{j}\left(T_{j}\right) .
$$

Thus, we have the following result.

Theorem 6 (Bloch [5]). The elliptic dilogarithm $D^{E}$ extends by linearity to a map from $\mathbb{Z}[E(\mathbb{Q})]^{-}$to $\mathbb{C}$. Let $x, y \in \mathbb{Q}(E)$ and $\{x, y\} \in K_{2}(E)$. Then

$$
r(\{x, y\})[\gamma]=D^{E}((x) \diamond(y)),
$$

where $\gamma$ is a generator of $H_{1}(E, \mathbb{Z})^{-}$. 
Remark 7. The result above implies in particular that

$$
D^{E}((x) \diamond(1-x))=0
$$

for any $x \in \mathbb{Q}(E)$.

Deninger [11] was the first to write a formula of the form

$$
\mathrm{m}(P)=\frac{1}{2 \pi} r(\{x, y\})[\gamma] .
$$

Then Rodriguez-Villegas [17] made a thorough study of the properties of $\eta(x, y)$ and combined the above expression with Theorem 6 to deduce identity (1.2) in [18].

We will now give some more details about (2.4). Let $P(x, y) \in \mathbb{C}[x, y]$ be a polynomial of degree 2 on $y$. We may then write

$$
P(x, y)=P^{*}(x)\left(y-y_{1}(x)\right)\left(y-y_{2}(x)\right),
$$

where $y_{1}(x), y_{2}(x)$ are algebraic functions.

By applying Jensen's formula with respect to the variable $y$, we have

$$
\begin{aligned}
\mathrm{m}(P)-\mathrm{m}\left(P^{*}\right)= & \frac{1}{(2 \pi i)^{2}} \int_{\mathbb{T}^{2}} \log |P(x, y)| \frac{d x}{x} \frac{d y}{y}-\mathrm{m}\left(P^{*}\right) \\
= & \frac{1}{(2 \pi i)^{2}} \int_{\mathbb{T}^{2}}\left(\log \left|y-y_{1}(x)\right|+\log \left|y-y_{2}(x)\right|\right) \frac{d x}{x} \frac{d y}{y} \\
= & \frac{1}{2 \pi i} \int_{|x|=1,\left|y_{1}(x)\right| \geq 1} \log \left|y_{1}(x)\right| \frac{d x}{x} \\
& +\frac{1}{2 \pi i} \int_{|x|=1,\left|y_{2}(x)\right| \geq 1} \log \left|y_{2}(x)\right| \frac{d x}{x} .
\end{aligned}
$$

Now suppose that $\left|y_{2}(x)\right| \leq 1$ as long as $|x|=1$ (this happens, for instance, if the constant coefficient of $P \in \mathbb{C}[x][y]$ is 1$)$. Then the above formula may be rewritten as

$$
\begin{aligned}
\mathrm{m}(P)-\mathrm{m}\left(P^{*}\right) & =\frac{1}{2 \pi i} \int_{|x|=1,\left|y_{1}(x)\right| \geq 1} \log \left|y_{1}(x)\right| \frac{d x}{x} \\
& =-\frac{1}{2 \pi} \int_{|x|=1,\left|y_{1}(x)\right| \geq 1} \eta\left(x, y_{1}\right) .
\end{aligned}
$$

When $P$ corresponds to an elliptic curve and when the set $\left\{|x|=1,\left|y_{1}(x)\right| \geq 1\right\}$ can be seen as a cycle in $H_{1}(E, \mathbb{Z})^{-}$, then we may be able to recover a formula of the type (2.4). This has to be examined on a case-by-case basis.

\section{Curves, Points, and an Isomorphism}

The first step in proving Theorem 1 consists of identifying both sides of Eq. (1.5) with elliptic curves.

A recurrent condition in Sec. 2 is that both the curve and the rational functions over the curve be defined over $\mathbb{Q}$. It is discussed in $[7$, p. 54] that the right-hand 
side of Eq. (1.5) is given by

$$
\mathrm{m}\left(P_{i}(x, y)\right)=\mathrm{m}\left(x_{0}-\frac{1}{x_{0}}+y_{0}-\frac{1}{y_{0}}+1\right) .
$$

To see this, it suffices to consider the change of variables $x=i x_{0}$ and $y=i y_{0}$ and divide by $i$, which does not affect the Mahler measure.

Remark 8. From now on, we will work with the polynomial in the right-hand side of the above equation, which we will denote by $R_{1}(x, y)$. We will also rename by $x, y$ the functions $x_{0}, y_{0}$.

Let $E$ be the elliptic curve defined by $\left\{F_{3}=0\right\}$. We have the following change of variables (see $[7,15])$ :

$$
\varphi: R_{1}: x-\frac{1}{x}+y-\frac{1}{y}+1=0 \rightarrow E: Y^{2}+3 X Y=X^{3}+X
$$

given by

$$
\begin{gathered}
X=-x y, \quad Y=x(1+y-x y), \\
x=\frac{Y+X}{X+1}, \quad y=-\frac{Y+2 X}{X+1} .
\end{gathered}
$$

The curve $E$ has rational torsion $E(\mathbb{Q})_{\text {tors }}=\langle P\rangle \cong \mathbb{Z} / 4 \mathbb{Z}$, where $P=(-1,2)$, $2 P=(0,0)$, and $3 P=-P=(-1,1) . E$ has also some notable complex points

$$
A=(i, 0), \quad \bar{A}=(-i, 0) \text { satisfying } A+\bar{A}=2 P .
$$

We will see in Sec. 5 that the points $A, \bar{A}$ have infinite order.

\section{The Diamond Operation}

In this section we proceed to compute the diamond operation for the pairs of functions $x \circ \varphi^{-1}, y \circ \varphi^{-1}$ and $X, Y \in \mathbb{Q}(E)$. Then we will compare these two results.

Proposition 9. We have the following relations in $\mathbb{Z}[E(\mathbb{Q})]^{-}$:

$$
(X) \diamond(Y)=4(A)+4(\bar{A})
$$

and

$$
\left(x \circ \varphi^{-1}\right) \diamond\left(y \circ \varphi^{-1}\right)=8(P) .
$$

Proof. By looking first at the divisors of some rational functions on $E$, we have

$$
\begin{aligned}
(X) & =2(2 P)-2 O, \\
(X+1) & =(P)+(-P)-2 O, \\
(Y+2 X) & =2(P)+(2 P)-3 O, \\
(Y+X) & =2(-P)+(2 P)-3 O, \\
(Y) & =(2 P)+(A)+(\bar{A})-3 O .
\end{aligned}
$$


If we apply the diamond operation between the first and the last of the above equations, we immediately obtain (4.1):

$$
(X) \diamond(Y)=4(A)+4(\bar{A}) .
$$

By composing with $\varphi^{-1}$, we have

$$
\begin{aligned}
& \left(x \circ \varphi^{-1}\right)=-(P)+(2 P)+(-P)-O, \\
& \left(y \circ \varphi^{-1}\right)=(P)+(2 P)-(-P)-O .
\end{aligned}
$$

We obtain (4.2) by applying the diamond operation.

We need to relate the dilogarithm $D^{E}$ evaluated in both elements $\left(x \circ \varphi^{-1}\right) \diamond$ $\left(y \circ \varphi^{-1}\right)$ and $(X) \diamond(Y)$ of $\mathbb{Z}[E(\mathbb{C})]^{-}$. For two elements $\alpha, \beta \in \mathbb{Z}[E(\mathbb{C})]^{-}$, we will write

$$
\alpha \sim \beta
$$

when

$$
D^{E}(\alpha)=D^{E}(\beta)
$$

In particular, Remark 7 implies that $\alpha \sim \beta$ if

$$
\alpha-\beta=\sum c_{i}\left(f_{i}\right) \diamond\left(1-f_{i}\right) \quad \text { for some } f_{i} \in \mathbb{Q}(E), c_{i} \in \mathbb{Z} \text {. }
$$

Proposition 10. We have the following relationship:

$$
4(X) \diamond(Y) \sim-7\left(x \circ \varphi^{-1}\right) \diamond\left(y \circ \varphi^{-1}\right) .
$$

Proof. Our goal is to find rational functions $f_{i} \in \mathbb{Q}(E)$ such that we can write Eq. (4.3) for $\alpha$ and $\beta$ multiples of $\left(x \circ \varphi^{-1}\right) \diamond\left(y \circ \varphi^{-1}\right)$ and $(X) \diamond(Y)$, respectively.

We start by considering $f_{1}$ to be the line passing through $A$ and $\bar{A}$ so that $1-f_{1}$ passes through the point $-P$. We obtain

$$
\begin{aligned}
(Y) & =(2 P)+(A)+(\bar{A})-3 O, \\
(1-Y) & =(-P)+(U)+\left(U^{\sigma}\right)-3 O,
\end{aligned}
$$

where $U=\left(\frac{1+\sqrt{5}}{2}, 1\right)$ and $U^{\sigma}=\left(\frac{1-\sqrt{5}}{2}, 1\right)$. Here $\sigma$ is the Galois element $\sqrt{5} \rightarrow$ $-\sqrt{5}$. These new points satisfy $U+U^{\sigma}=P$.

Now we compute the diamond operation of the previous functions:

$$
\begin{aligned}
0 \sim & (Y) \diamond(1-Y) \\
\sim & -4(P)-3(A)-3(\bar{A})+3(U)+3\left(U^{\sigma}\right)+(P+U)+\left(P+U^{\sigma}\right) \\
& +(A-U)+(\bar{A}-U)+\left(A-U^{\sigma}\right)+\left(\bar{A}-U^{\sigma}\right) .
\end{aligned}
$$


Next, we consider $f_{2}$ to be the tangent line at $-P$ so that $1-f_{2}$ passes through $P+U$ and $P+U^{\sigma}$. We have

$$
\begin{aligned}
(Y+X) & =2(-P)+(2 P)-3 O, \\
(1-Y-X) & =(P)+(P+U)+\left(P+U^{\sigma}\right)-3 O,
\end{aligned}
$$

where $P+U=\left(\frac{-1-\sqrt{5}}{2}, \frac{3+\sqrt{5}}{2}\right)$ and $P+U^{\sigma}=\left(\frac{-1+\sqrt{5}}{2}, \frac{3-\sqrt{5}}{2}\right)$.

Once again, we find the diamond operation of the previous functions,

$$
\begin{aligned}
0 & \sim(Y+X) \diamond(1-Y-X) \\
& \sim 10(P)+(U)+\left(U^{\sigma}\right)+5(P+U)+5\left(P+U^{\sigma}\right) .
\end{aligned}
$$

Now we find a quadratic polynomial $f_{3}$ whose divisor is supported in $\langle P\rangle, P+U$ and $P+U^{\sigma}$, and such that the divisor of $1-f_{3}$ is supported in $P, A$ and $\bar{A}$.

$$
\begin{aligned}
\left(Y-X^{2}\right) & =(2 P)+(-P)+(P+U)+\left(P+U^{\sigma}\right)-4 O, \\
\left(1-Y+X^{2}\right) & =2(P)+(A)+(\bar{A})-4 O .
\end{aligned}
$$

We compute the diamond operation and obtain

$$
\begin{aligned}
0 \sim & \left(Y-X^{2}\right) \diamond\left(1-Y+X^{2}\right) \\
\sim & 14(P)+5(A)+5(\bar{A})+2(U)+2\left(U^{\sigma}\right)-4(P+U)-4\left(P+U^{\sigma}\right) \\
& +(A-U)+(\bar{A}-U)+\left(A-U^{\sigma}\right)+\left(\bar{A}-U^{\sigma}\right) .
\end{aligned}
$$

We combine Eqs. (4.4)-(4.6) and obtain

$$
\begin{aligned}
0 & \sim-(Y) \diamond(1-Y)+(Y-X) \diamond(1-Y+X)+\left(Y-X^{2}\right) \diamond\left(1-Y+X^{2}\right) \\
& \sim 28(P)+8(A)+8(\bar{A}) .
\end{aligned}
$$

In other words, we have proven that

$$
7 D^{E}(P)+2 D^{E}(A)+2 D^{E}(\bar{A})=0 .
$$

Combining the line above with Eqs. (4.2) and (4.1), we finally reach the desired relationship.

\section{An Interesting Case of Beřlinson's Conjectures}

The goal of this section is to further reflect on the meaning of Eq. (4.7) in the context of Bellinson's conjectures. The results of this section are not needed for the proof of Theorem 1 but they are interesting in their own right.

We will start by proving that $D^{E}(A)=D^{E}(\bar{A})$ and then showing that $A$ is non-torsion. The first property is consequence of the following more general fact.

Lemma 11. Let $E / \mathbb{R}$ be an elliptic curve. Let $B=(i b, \beta) \in E(\mathbb{C})$ with $b \in \mathbb{R}$ and let $\bar{B}=(-i b, \bar{\beta})$ be the point resulting from conjugating each coordinate of $B$. Then

$$
D^{E}(B)=D^{E}(\bar{B})
$$

where $D^{E}$ is the elliptic dilogarithm given by (2.3). 
Proof. We revisit diagram (2.2) and write for $\Lambda=\mathbb{Z}+\tau \mathbb{Z}$,

$$
\mathcal{F}: E(\mathbb{C}) \rightarrow \mathbb{C} / \Lambda
$$

the map given by

$$
S \rightarrow \frac{1}{\Omega} \int_{O}^{S} \omega \bmod \Lambda,
$$

where $\Omega$ is the real period and $\omega$ is the standard invariant differential.

Thus,

$$
\mathcal{F}\left(\wp(u), \wp^{\prime}(u)\right) \equiv u \quad \bmod \Lambda
$$

Let $Y^{2}+a_{1} X Y+a_{3} Y=X^{3}+a_{2} X^{2}+a_{4} X+a_{6}$ be the Weierstrass form for $E$ and write

$$
f(X)=\left(a_{1} X+a_{3}\right)^{2}+4\left(X^{3}+a_{2} X^{2}+a_{4} X+a_{6}\right) .
$$

Then the standard invariant differential is given by

$$
\omega=\frac{d X}{\sqrt{f(X)}} .
$$

By computing $\mathcal{F}(B)$ and $\mathcal{F}(\bar{B})$, we obtain

$$
\begin{aligned}
& \Omega \mathcal{F}(B)=\int_{O}^{B} \omega=-\int_{i b}^{i \infty} \frac{d X}{\sqrt{f(X)}}=-\int_{b}^{\infty} \frac{d i Z}{\sqrt{f(i Z)}} \\
& \Omega \mathcal{F}(\bar{B})=\int_{O}^{\bar{B}} \omega=-\int_{-i b}^{-i \infty} \frac{d X}{\sqrt{f(X)}}=-\int_{b}^{\infty} \frac{-d i Z}{\sqrt{f(-i Z)}},
\end{aligned}
$$

and we conclude that $\overline{\mathcal{F}(B)}=\mathcal{F}(\bar{B})$.

We proceed to compute the elliptic dilogarithm in $B$. By (2.3), we have

$$
\begin{aligned}
D^{E}(B) & =\sum_{n \in \mathbb{Z}} D\left(q^{n} z_{B}\right)=\sum_{n \in \mathbb{Z}} D\left(e^{2 \pi i(n \tau+\mathcal{F}(B))}\right) \\
& =\sum_{n \in \mathbb{Z}} D\left(e^{2 \pi i(n \tau+\overline{\mathcal{F}(\bar{B})})}\right) \\
& =\sum_{n \in \mathbb{Z}} D\left(\overline{\left.e^{-2 \pi i(n \bar{\tau}+\mathcal{F}(\bar{B}))}\right)}\right.
\end{aligned}
$$

Recall that $D(x)=-D\left(x^{-1}\right)=-D(\bar{x})$ (see, for example, [22, pp. 10-11]). Thus, 


$$
\begin{aligned}
D^{E}(B) & =-\sum_{n \in \mathbb{Z}} D\left(e^{-2 \pi i(n \bar{\tau}+\mathcal{F}(\bar{B}))}\right) \\
& =\sum_{n \in \mathbb{Z}} D\left(e^{2 \pi i(n \bar{\tau}+\mathcal{F}(\bar{B}))}\right) \\
& =\sum_{n \in \mathbb{Z}} D\left(e^{2 \pi i(-n \bar{\tau}+\mathcal{F}(\bar{B}))}\right) \\
& =\sum_{n \in \mathbb{Z}} D\left(e^{2 \pi i(n(-\bar{\tau})+\mathcal{F}(\bar{B}))}\right) \\
& =D^{E}(\bar{B})
\end{aligned}
$$

since $\Lambda=\bar{\Lambda}=\mathbb{Z}+(-\bar{\tau}) \mathbb{Z}$ as the curve is defined over the reals.

By setting $A=(i, 0)$ and considering $E: Y^{2}+3 X Y=X^{3}+X$ in the previous lemma, we obtain that $D^{E}(A)=D^{E}(\bar{A})$.

Lemma 12. $A=(i, 0) \in E: Y^{2}+3 X Y=X^{3}+X$ is non-torsion.

Proof. Consider the isomorphism

$$
\phi: E \rightarrow E_{1}: Y_{1}^{2}=X_{1}^{3}-9 X_{1}^{2}+16 X_{1}
$$

given by

$$
Y_{1}=4 i(2 Y+3 X), \quad X_{1}=-4 X
$$

The image of $A$ is $A_{1}=(-4 i,-12)$ with $2 A_{1}=\left(\frac{16}{9},-\frac{64}{27}\right)$. By Nagell-Lutz theorem, $2 A_{1}$ is non-torsion, and it means that the same is true for $2 A$ and for $A$.

Thus we have been able to write Eq. (4.7) as

$$
7 D^{E}(P)=-4 D^{E}(A) .
$$

The above equation is very interesting because it relates the dilogarithm evaluated in the rational torsion point $P$ with the dilogarithm evaluated in a complex point of infinite order $A$.

We recall that the Bloch and Bellinson conjectures predict for $E / \mathbb{Q}$ that

$$
L(E, 2)=\frac{\pi}{N} D^{E}(\alpha) \quad \text { for some } \alpha \in \mathbb{Z}\left[E(\overline{\mathbb{Q}})_{\text {tors }}\right],
$$

where $N$ is the conductor of $E$. This was proven for CM elliptic curves by Bloch [5] and for elliptic modular curves by Bělinson [4]. Zagier and Gangl [23] further conjectured that

$$
L(E, 2)=\frac{\pi}{N} D^{E}(\alpha) \quad \text { for some } \alpha \in \mathbb{Z}[E(\overline{\mathbb{Q}})]^{\mathrm{Gal}(\overline{\mathbb{Q}} / \mathbb{Q})} .
$$


It can be seen from Eqs. (1.6) and (4.2) and the functional equation of $L\left(E_{17}, s\right)$ that

$$
L\left(E_{17}, 2\right)=\frac{8 \pi}{17} D^{E}(P) .
$$

Combining the equation above with (5.1) we obtain that

$$
L\left(E_{17}, 2\right)=-\frac{32 \pi}{119} D^{E}(A) .
$$

This identity is very interesting as it does not fit the previous versions of the Bloch and Běllinson's conjecture.

\section{The Homology Class of the Integration Path}

In this section we identify the integration path in the Mahler measure formulas involved in Eq. (1.5) in terms of homology classes in the elliptic curve.

Following the discussion at the end of Sec. 2 and Remark 8, we need to identify the integration paths for the Mahler measures of $R_{1}$ and $F_{3}$. A starting point is to look for the intersection of each of the curves $\left\{R_{1}=0\right\}$ and $\left\{F_{3}=0\right\}$ with the unit torus $\mathbb{T}^{2}$. This will give an idea if there is a root $y(x)$ whose absolute value remains always larger or smaller than 1 as $|x|=1$.

For the case of $R_{1}$, since $x-\frac{1}{x}, y-\frac{1}{y} \in i \mathbb{R}$ for $|x|=|y|=1$, we can never have $R_{1}(x, y)=0$ in $\mathbb{T}^{2}$.

If we think of $y R_{1}(x, y) \in \mathbb{C}(x)[y]$ and use the equation to implicitly define $y$ as a function of $x$, we obtain two roots $y_{1}(x)$ and $y_{2}(x)$ that satisfy $y_{1}(x) y_{2}(x)=-1$ and they never satisfy $\left|y_{j}(x)\right|=1$ as long as $|x|=1$. Therefore one of them, say $y_{1}(x)$, has always absolute value larger than 1 and the other, say $y_{2}(x)$, smaller. We can summarize by saying that the integration set for the Mahler measure of $R_{1}$ is given by

$$
\gamma_{1}=\left\{\left(x, y_{1}\right):|x|=1\right\}
$$

For the second curve, we may make the change of variables $Y=Z X$ and divide by $X^{2}$. Thus the Mahler measure of $F_{3}$ equals that of

$$
G(X, Z)=Z^{2}+3 Z-X-\frac{1}{X} .
$$

Suppose that $|X|=1$ and write $X=e^{i \theta}$. Then we need to solve

$$
Z^{2}+3 Z-2 \cos \theta=0
$$

and

$$
Z_{ \pm}=\frac{-3 \pm \sqrt{9+8 \cos (\theta)}}{2}
$$

The interior of the square root is always $\geq 1$, therefore the roots are always real. In addition, notice that $9+8 \cos (\theta) \leq 17$ and $\sqrt{17}<5$. Therefore, $\left|Z_{+}\right|<1$. As for 
$Z_{-}$, we have that the only possibility for $\left|Z_{-}\right|=1$ is that $Z_{-}= \pm 1$. But the only solution to

$$
1+3-2 \cos (\theta)=0 \quad \text { or } \quad 1-3-2 \cos (\theta)=0
$$

is with $Z_{-}=-1$ and $\theta=\pi$.

Outside of $Z_{-}=-1$, we have that $\left|Z_{-}\right|>1$ as long as $|X|=1$. Thus, the integration cycle for the Mahler measure of $G$ is given by

$$
\gamma_{2}=\left\{\left(X, Z_{-}\right):|X|=1\right\}
$$

To summarize, we have proven the following result.

Proposition 13. Let $y_{1}=y_{1}(x)$ be the root of $R_{1}=0$ such that $\left|y_{1}\right|>1$ as long as $|x|=1$. Let $Z_{-}=Z_{-}(X)$ be the root of $G=0$ such that $\left|Z_{-}\right| \geq 1$ as long as $|X|=1$. Then we have

$$
\begin{aligned}
& \mathrm{m}\left(P_{i}\right)=\mathrm{m}\left(R_{1}\right)=-\frac{1}{2 \pi} \int_{|x|=1} \eta\left(x, y_{1}\right), \\
& \mathrm{m}\left(F_{3}\right)=\mathrm{m}(G)=-\frac{1}{2 \pi} \int_{|X|=1} \eta\left(X, Z_{-}\right) .
\end{aligned}
$$

Remark 14. Because the polynomials are tempered (see [17]), the integrals above only depend on the homology classes of $\varphi\left(\gamma_{1}\right)$ and $\gamma_{2}$ in $H_{1}(E, \mathbb{Z})$.

We need to determine the homology class of the integration cycles in $H_{1}(E, \mathbb{Z})$.

Proposition 15. Let $\gamma_{1}$ and $\gamma_{2}$ be given by Eqs. (6.1) and (6.2), respectively. Then $\varphi_{*}\left[\gamma_{1}\right],\left[\gamma_{2}\right] \in H_{1}(E, \mathbb{Z})^{-}$and $\pm \varphi_{*}\left[\gamma_{1}\right]=\left[\gamma_{2}\right]$ in $H_{1}(E, \mathbb{Z})^{-}$.

Remark 16. Since we know that the Mahler measure is always positive, the sign ambiguity is not a problem here.

Before continuing we need to prove an identity between elliptic integrals that will be key to the proof of Proposition 15.

Lemma 17. We have that

$$
\int_{-\pi}^{\pi} \frac{d \theta}{\sqrt{4+(2 i \sin \theta+1)^{2}}}=\int_{-\pi}^{\pi} \frac{d \theta}{\sqrt{9+8 \cos \theta}} .
$$

Proof. It should be noted that the left-hand side of (6.3) is real. Indeed, separating the integration domain in two pieces and making the substitution $\tau=-\theta$,

$$
\begin{aligned}
\int_{-\pi}^{\pi} \frac{d \theta}{\sqrt{4+(2 i \sin \theta+1)^{2}}} & =\int_{0}^{\pi} \frac{d \theta}{\sqrt{4+(2 i \sin \theta+1)^{2}}}+\int_{-\pi}^{0} \frac{d \theta}{\sqrt{4+(2 i \sin \theta+1)^{2}}} \\
& =\int_{0}^{\pi} \frac{d \theta}{\sqrt{4+(2 i \sin \theta+1)^{2}}}+\int_{0}^{\pi} \frac{d \tau}{\sqrt{4+(-2 i \sin \tau+1)^{2}}} .
\end{aligned}
$$


Thus,

$$
\int_{-\pi}^{\pi} \frac{d \theta}{\sqrt{4+(2 i \sin \theta+1)^{2}}} \in \mathbb{R} .
$$

In what follows, we proceed to write both integrals from Eq. (6.3) in terms of Complete Elliptic Integrals of the First Kind. We continue working with the lefthand side. Let $t=\sin \theta$ for $0 \leq \theta \leq \frac{\pi}{2}$. Then $\frac{d t}{\sqrt{1-t^{2}}}=d \theta$ and we have

$$
\begin{aligned}
\int_{-\pi}^{\pi} \frac{d \theta}{\sqrt{4+(2 i \sin \theta+1)^{2}}} & =4 \operatorname{Re} \int_{0}^{\frac{\pi}{2}} \frac{d \theta}{\sqrt{4+(2 i \sin \theta+1)^{2}}} \\
& =4 \operatorname{Re} \int_{0}^{1} \frac{d t}{\sqrt{\left(1-t^{2}\right)\left(4+(2 i t+1)^{2}\right)}}
\end{aligned}
$$

Consider the following change of variables

$$
z=\frac{1-i\left(\frac{1+\sqrt{17}}{4}\right) t}{t-i\left(\frac{1+\sqrt{17}}{4}\right)}, \quad t=\frac{1+i\left(\frac{1+\sqrt{17}}{4}\right) z}{z+i\left(\frac{1+\sqrt{17}}{4}\right)} .
$$

The relationship between the differentials is given by

$$
d t=-\left(\frac{17+\sqrt{17}}{8}\right) \frac{d z}{\left(z+i\left(\frac{1+\sqrt{17}}{4}\right)\right)^{2}} .
$$

Applying this change of variables, we obtain

$$
\begin{aligned}
\int_{-\pi}^{\pi} & \frac{d \theta}{\sqrt{4+(2 i \sin \theta+1)^{2}}} \\
& =4 \operatorname{Re} \int_{0}^{1} \frac{d t}{\sqrt{\left(1-t^{2}\right)\left(4+(2 i t+1)^{2}\right)}} \\
& =\left(\frac{-1+\sqrt{17}}{2}\right) \operatorname{Re} \int_{i\left(\frac{-1+\sqrt{17}}{4}\right)}^{1} \frac{d z}{\sqrt{\left(1-z^{2}\right)\left(1-\left(\frac{-1+\sqrt{17}}{1+\sqrt{17}}\right)^{2} z^{2}\right)}}
\end{aligned}
$$

We complete the integration path with the imaginary axis connecting 0 to $i\left(\frac{-1+\sqrt{17}}{4}\right)$ and the real axis connecting 0 to 1 . Since the interior of the region is contained in the unit disk, the integrand has a holomorphic branch and the integral around the whole boundary equals zero. 
The integral

$$
\int_{0}^{i\left(\frac{-1+\sqrt{17}}{4}\right)} \frac{d z}{\sqrt{\left(1-z^{2}\right)\left(1-\left(\frac{-1+\sqrt{17}}{1+\sqrt{17}}\right)^{2} z^{2}\right)}} \in i \mathbb{R},
$$

since the domain is included in the imaginary axis.

Therefore, we conclude that

$$
\begin{aligned}
\int_{-\pi}^{\pi} \frac{d \theta}{\sqrt{4+(2 i \sin \theta+1)^{2}}} & =\left(\frac{-1+\sqrt{17}}{2}\right) \int_{0}^{1} \frac{d z}{\sqrt{\left(1-z^{2}\right)\left(1-\left(\frac{-1+\sqrt{17}}{1+\sqrt{17}}\right)^{2} z^{2}\right)}} \\
& =\left(\frac{-1+\sqrt{17}}{2}\right) K\left(\frac{-1+\sqrt{17}}{1+\sqrt{17}}\right),
\end{aligned}
$$

where $K(k)$ denotes the Complete Elliptic Integral of the First Kind

$$
K(k):=\int_{0}^{\frac{\pi}{2}} \frac{d \theta}{\sqrt{1-k^{2} \sin ^{2} \theta}}=\int_{0}^{1} \frac{d t}{\sqrt{\left(1-t^{2}\right)\left(1-k^{2} t^{2}\right)}} .
$$

Now we focus on the right-hand side of Eq. (6.3). By setting $\theta=2 \tau$, we have

$$
\begin{aligned}
\int_{-\pi}^{\pi} \frac{d \theta}{\sqrt{9+8 \cos \theta}} & =2 \int_{0}^{\pi} \frac{d \theta}{\sqrt{9+8 \cos \theta}} \\
& =4 \int_{0}^{\frac{\pi}{2}} \frac{d \tau}{\sqrt{17-16 \sin ^{2} \tau}} \\
& =\frac{4}{\sqrt{17}} K\left(\frac{4}{\sqrt{17}}\right) .
\end{aligned}
$$

Recall the following identity, which can be deduced from the arithmetic--geometric mean identity:

$$
\frac{1}{a} K\left(\frac{\sqrt{a^{2}-b^{2}}}{a}\right)=\frac{2}{a+b} K\left(\frac{a-b}{a+b}\right) .
$$

Setting $a=\sqrt{17}, b=1$ above, we obtain

$$
\frac{1}{\sqrt{17}} K\left(\frac{4}{\sqrt{17}}\right)=\frac{2}{1+\sqrt{17}} K\left(\frac{\sqrt{17}-1}{\sqrt{17}+1}\right) .
$$

This concludes the proof of Eq. (6.3).

Proof of Proposition 15. Our goal is to see that

$$
\pm \int_{\varphi_{*}(|x|=1)} \omega=\int_{|X|=1} \omega \in i \mathbb{R} .
$$


Over $E$, we have

$$
\omega=\frac{d X}{2 Y+3 X}
$$

We would like to find $\varphi^{*} \omega$, namely, to express $\omega$ in terms of $x, y$ so that we can integrate it over $\gamma_{1}$. To begin, by looking at the formulas for $\varphi$, we have

$$
d X=-(x d y+y d x) .
$$

By differentiating $R_{1}$ we get

$$
\left(1+\frac{1}{x^{2}}\right) d x+\left(1+\frac{1}{y^{2}}\right) d y=0 .
$$

Then, we obtain

$$
d X=\frac{\left(x+\frac{1}{x}-y-\frac{1}{y}\right) d x}{\left(1+\frac{1}{y^{2}}\right)}
$$

On the other hand,

$$
2 Y+3 X=x(2-y-2 x y) .
$$

Finally, combining (6.5) and (6.6) with (6.4), we obtain

$$
\varphi^{*} \omega=\frac{\left(x+\frac{1}{x}-y-\frac{1}{y}\right) d x}{x\left(y+\frac{1}{y}\right)\left(\frac{2}{y}-1-2 x\right)} .
$$

Thus, our goal is to integrate

$$
\int_{\varphi_{*}(|x|=1)} \omega=\int_{|x|=1} \varphi^{*} \omega=\int_{|x|=1} \frac{\left(x+\frac{1}{x}-y-\frac{1}{y}\right) d x}{x\left(y+\frac{1}{y}\right)\left(\frac{2}{y}-1-2 x\right)},
$$

where $y$ is the root of absolute value $>1$ in $x-\frac{1}{x}+y-\frac{1}{y}+1=0$.

Let $x=e^{i \theta}$ with $-\pi \leq \theta \leq \pi$. The roots of the equation are given by

$$
\begin{aligned}
y_{ \pm} & =\frac{-\left(x-\frac{1}{x}+1\right) \pm \sqrt{\left(x-\frac{1}{x}+1\right)^{2}+4}}{2} \\
& =\frac{-(2 i \sin \theta+1) \pm \sqrt{(2 i \sin \theta+1)^{2}+4}}{2} .
\end{aligned}
$$

For the sake of notation, we write

$$
y_{ \pm}=\frac{-(2 i \sin \theta+1) \pm \sqrt{\Delta}}{2} .
$$


Also, remark that $y_{+} y_{-}=-1$ from the equation $R_{1}=0$.

With this change of variables, we have

$$
\frac{2}{y_{ \pm}}-1-2 x=-\left(2 y_{\mp}+1+2 x\right)=-2 \cos \theta \pm \sqrt{\Delta} .
$$

Taking the above into account, we obtain for $\varphi^{*} \omega$

$$
\begin{aligned}
\varphi^{*} \omega & =\frac{\left(x+\frac{1}{x}-y_{ \pm}-\frac{1}{y_{ \pm}}\right) d x}{x\left(y_{ \pm}+\frac{1}{y_{ \pm}}\right)\left(\frac{2}{y_{ \pm}}-1-2 x\right)} \\
& =\frac{(2 \cos \theta \mp \sqrt{\Delta})}{ \pm \sqrt{\Delta}(-2 \cos \theta \pm \sqrt{\Delta})} i d \theta \\
& =\mp \frac{i d \theta}{\sqrt{\Delta}} \\
& =\mp \frac{i d \theta}{\sqrt{(2 i \sin \theta+1)^{2}+4}} .
\end{aligned}
$$

In sum, (6.7) becomes

$$
\int_{\varphi_{*}(|x|=1)} \omega=\mp i \int_{-\pi}^{\pi} \frac{d \theta}{\sqrt{4+(2 i \sin \theta+1)^{2}}},
$$

and the sign depends on the choice of $y_{ \pm}$.

On the other hand, we need to evaluate

$$
\int_{|X|=1} \omega=\int_{|X|=1} \frac{d X}{2 Y+3 X}
$$

where $Y$ is the root of absolute value $>1$.

Again with the change of variables $X=e^{i \theta}$, we have

$$
\begin{aligned}
Y_{ \pm} & =\frac{-3 X \pm \sqrt{9 X^{2}+4\left(X^{3}+X\right)}}{2} \\
& =X\left(\frac{-3 \pm \sqrt{9+8 \cos \theta}}{2}\right) .
\end{aligned}
$$

Referring to the discussion about $G(X, Z)$, the path that we must take corresponds to the negative sign. Thus, in the integral (6.9), we will take

$$
Y=X\left(\frac{-3-\sqrt{9+8 \cos \theta}}{2}\right) \text {. }
$$

For the expression for $\omega$, we obtain

$$
\begin{aligned}
\omega & =\frac{d X}{2 Y+3 X} \\
& =-\frac{i d \theta}{\sqrt{9+8 \cos \theta}} .
\end{aligned}
$$


Finally, Eq. (6.9) becomes

$$
\int_{|X|=1} \omega=-i \int_{-\pi}^{\pi} \frac{d \theta}{\sqrt{9+8 \cos \theta}} .
$$

Since the right-hand side is purely imaginary, we conclude that $\left[\gamma_{2}\right] \in H_{1}(E, \mathbb{Z})^{-}$.

By combining Eqs. (6.8) and (6.10), the result follows from Lemma 17.

\section{The End of the Proof}

Here we finish the proof of Theorem 1. Recall that Proposition 13 gives

$$
\begin{aligned}
& \mathrm{m}\left(P_{i}\right)=-\frac{1}{2 \pi} \int_{\gamma_{1}} \eta(x, y), \\
& \mathrm{m}\left(F_{3}\right)=-\frac{1}{2 \pi} \int_{\gamma_{2}} \eta(X, Y),
\end{aligned}
$$

where we have used the fact that $Z X=Y$, the multiplicativity of $\eta$, and the fact that $\eta(X, X)=0$.

Proposition 15 implies that $\pm \varphi_{*}\left[\gamma_{1}\right]=\left[\gamma_{2}\right] \in H_{1}(E, \mathbb{Z})^{-}$and $\pm \varphi_{*}\left[\gamma_{1}\right]=\left[\gamma_{2}\right]=$ $C[\gamma] \in H_{1}(E, \mathbb{Z})^{-}$, where we have denoted by $[\gamma]$ a generator of $H_{1}(E, \mathbb{Z})^{-}$. By Theorem 6 , we have

$$
\begin{gathered}
\int_{\gamma_{1}} \eta(x, y)=\int_{\varphi_{*}\left(\gamma_{1}\right)} \eta\left(x \circ \varphi^{-1}, y \circ \varphi^{-1}\right)= \pm C D^{E}\left(\left(x \circ \varphi^{-1}\right) \diamond\left(y \circ \varphi^{-1}\right)\right), \\
\int_{\gamma_{2}} \eta(X, Y)=C D^{E}((X) \diamond(Y)) .
\end{gathered}
$$

Now, combining with Proposition 10, we conclude the proof of Theorem 1.

\section{Conclusion}

We have proven a new formula for the Mahler measure of a Weierstrass form. This particular polynomial seems to be the first formula to be proven in the family $F_{k}$. Boyd [7] actually considers $F_{k}$ as part of a two-parameter family for which he finds

$$
\mathrm{m}\left(y^{2}+k x y-x^{3}-b x\right) \stackrel{?}{=} \frac{1}{4} \log |b|+r L^{\prime}(E, 0) .
$$

The first term in the right-hand side appears because the family is non-tempered unless $|b|=1$. It would be very interesting to prove other examples in this family when $|b| \neq 1$. This would require a careful study of tame symbols.

The only method that we know for finding relationships in $\mathbb{Z}[E(\mathbb{C})]^{-}$is the "parallel lines" method developed by Mellit in [16]. However, this method does not apply to what we did in Sec. 4. It would be desirable to develop a general method that includes our current work. 


\section{Acknowledgments}

We would like to thank Marie-José Bertin for her encouragement. We are also grateful to the referee for his/her helpful corrections. The authors were supported by the Natural Sciences and Engineering Research Council of Canada-NSERC (Discovery Grant 355412-2013 for ML, Undergraduate Student Research Awards for FR), and by the Fonds de la recherche du Québec - Nature et Technologies - FRQNT (Supplement of the NSERC Undergraduate Student Research Awards (BRPC) for FR).

\section{References}

[1] M.-J. Bertin, Mesure de Mahler et régulateur elliptique: preuve de deux relations "exotiques", in Number Theory, CRM Proceedings \& Lecture Notes, Vol. 36 (American Mathematical Society, Providence, RI, 2004), pp. 1-12.

[2] (2004) 175-188.

[3] A. A. Beĭlinson, Higher regulators and values of $L$-functions of curves, Funktsional. Anal. i Prilozhen. 14 (1980) 46-47.

[4] - Higher regulators of modular curves, in Applications of Algebraic K-theory to Algebraic Geometry and Number Theory, Part I, II, Contemporary Mathematics, Vol. 55 (American Mathematical Society, Providence RI, 1986), pp. 1-34.

[5] S. J. Bloch, Higher Regulators, Algebraic K-Theory, and Zeta Functions of Elliptic Curves, CRM Monograph Series, Vol. 11 (American Mathematical Society, Providence, RI, 2000).

[6] S. Bloch and D. Grayson, $K_{2}$ and $L$-functions of elliptic curves: computer calculations, in Applications of Algebraic K-Theory to Algebraic Geometry and Number Theory, Part I, II, Contemporary Mathematics, Vol. 55 (American Mathematical Society, Providence, RI, 1986), pp. 79-88.

[7] D. Boyd, Mahler's measure and special values of $L$-functions, Experiment. Math. 7 (1998) 37-82.

[8] F. Brunault, Étude de la valeur en $s=2$ de la fonction $L$ d'une courbe elliptique. Ph.D. thesis, Université Paris 7 Denis-Diderot (2005).

[9] Version explicite du théorème de Beylinson pour la courbe modulaire $X(N)$, C. R. Math. Acad. Sci. Paris 343(8) (2006) 505-510.

[10] , Regulators of Siegel units and applications, J. Number Theory 163 (2016) $542-569$.

[11] C. Deninger, Deligne periods of mixed motives, $K$-theory and the entropy of certain $\mathbb{Z}^{n}$-actions, J. Amer. Math. Soc. 10 (1997) 259-281.

[12] J. Guillera and M. D. Rogers, Mahler measure and the WZ algorithm, Proc. Amer. Math. Soc. 143(7) (2015) 2873-2886.

[13] M. N. Lalín, On a conjecture by Boyd, Int. J. Number Theory 6 (2010) 705-711.

[14] Mahler measure and elliptic curve $L$-functions at $s=3, J$. Reine Angew. Math. 709 (2015) 201-218.

[15] M. N. Lalín and M. D. Rogers, Functional equations for Mahler measures of genus-one curves, Algebra Number Theory 1(1) (2007) 87-117.

[16] A. Mellit, Elliptic dilogarithms and parallel lines, preprint (2012); arXiv:1207.4722 [math.NT].

[17] F. Rodriguez-Villegas, Modular Mahler measures I, in Topics in Number Theory, Mathematics and its Applications, Vol. 467 (Kluwer Academic Publishers, Dordrecht, 1999), pp. 17-48. 
[18] _ Identities between Mahler measures, in Number Theory for the Millennium, III (A K Peters, Natick, MA, 2002), pp. 223-229.

[19] M. Rogers and W. Zudilin, From $L$-series of elliptic curves to Mahler measures, Compositio Math. 148 (2012) 385-414.

[20] $ـ$ On the Mahler measure of $1+X+1 / X+Y+1 / Y$, Intern. Math. Res. Notices 2014 (2014) 2305-2326.

[21] N. Touafek and M. Kerada, Mahler measure and elliptic regulator: some identities. JP J. Algebra Number Theory Appl. 8(2) (2007) 271-285.

[22] D. Zagier, The dilogarithm function, in Frontiers in Number Theory, Physics, and Geometry. II (Springer, Berlin, 2007), pp. 3-65.

[23] D. Zagier and H. Gangl, Classical and elliptic polylogarithms and special values of $L$-series, in The Arithmetic and Geometry of Algebraic Cycles, NATO Science Series C: Mathematical and Physical Sciences, Vol. 548 (Kluwer Acadamic Publishers, Dordrecht, 2000), pp. 561-615.

[24] W. Zudilin, Regulator of modular units and Mahler measures, Math. Proc. Cambridge Philos. Soc. 156 (2014) 313-326. 